\title{
Evaluation of Infrared and Millimeter-wave Imaging Technologies Applied to Traffic Management
}

\author{
C. Arthur MacCarley \\ California Polytechnic State University \\ Brian M. Hemme \\ Loragen Corporation \\ Lawrence Klein \\ Consulting Engineer
}

Copyright @ 2000 Society of Automotive Engineers, Inc.

\begin{abstract}
This paper reviews the findings of a study commissioned by the California Department of Transportation to evaluate the potential of infrared and passive millimeterwave imaging technologies for traffic surveillance and automated detection. Images formed from radiation at infrared and millimeter wavelengths are characterized by fundamentally different information compared with visible-spectrum images, and generally improved ability to penetrate obscured atmospheres such as fog or dust.
\end{abstract}

Ten examples of commercially available infrared (IR) imaging technologies and one experimental passive millimeter-wave (MMW) radiometric imaging apparatus were evaluated with respect to the specific requirements of highway monitoring and traffic management. A suite of traffic images and performance-related test data were acquired for each imaging system over a range of traffic and environmental conditions. Evaluation criteria included the usable information content of images in both clear and obscured atmospheres, image noise, EIA NTSC standard video test metrics, advantages and limitations related to technical features, and human interface factors. A general metric was developed to facilitate the comparison of the quality and information content of the video images produced by imaging systems operating in different spectral bands. A software-based image processing program was developed to analyze digitized video samples produced by each imaging system with respect to this metric. Results are classified by spectral band, scene condition, and imaging technology. Recommendations are made regarding the attributes, and technical advantages and limitations of each imaging technology for specific traffic monitoring and management situations.

\section{INTRODUCTION}

The deployment of video cameras on roadways has improved both the accuracy and immediacy of the data available to traffic management center (TMC) personnel. Intelligent management actions may be engaged without the lag time associated with verbal reports or the limited information content associated with inductive loops or other simple vehicle detectors. Possible management actions include ramp meter rate control, driver advisement via Changeable Message Signs (CMS), and rapid deployment of emergency crews in response to traffic incidents or potential traffic hazards. Long-term data collection in support of improvements in facilities or management strategies is also enhanced.

Conventional video cameras, utilizing visible spectrum radiation for image formation, are adequate in most traffic surveillance situations. However, special situations may exist that warrant more sophisticated image sensing technologies, for example, conditions of limited visibility due to fog, dust, smoke, rain, glare or glint. Also, in darkness, only the headlights or tail lights of vehicles may be detectable. In the infrared (or IR) and millimeter-wave bands, solar or artificial illumination is not required for scene illumination. Blackbody radiation, especially in the longwave infrared band, is capable of providing "selfillumination" of vehicles on highways, especially when hot surfaces such as the engine or exhaust are exposed.

The information content of infrared or millimeter wave images is fundamentally different than that of visible spectrum images. This can be both and advantage and limitation, depending on the situation. Brightness in the medium and longwave infrared bands is better correlated with surface temperature than the reflective 
characteristics of surfaces in the scene. While this characteristic can be a source of confusion in human interpretation of IR images, it admits the possibility of non-intrusive thermal diagnostics, for example, the operational condition of brakes on heavy vehicles. Computer vision applications require consistent image characteristics independent of scene illumination, shadows, or image artifacts due to video saturation, such as vertical smear. Automated detection at night is often enhanced by or dependent upon the ability to discern the shape and features of complete vehicles, not just the headlights. This is of particular importance in the detection and interpretation of potentially hazardous traffic conditions, or the tracking of individual vehicles using optical flow techniques. Image data fusion, in which synthetic images formed and analyzed in real time from sensors operating in multiple spectral bands, may provide even more sophisticated and useful information for detection and traffic management decision-making.

Advanced image sensing methods utilize areas of the electromagnetic spectrum outside of the visible 0.4 to $0.7 \mu \mathrm{m}$ wavelength) range. Imaging technologies are available or are in development operating in the IR ( 0.8 to $14 \mu \mathrm{m}$ ) range, or in the microwave or millimeter-wave (35 to $94 \mathrm{GHz}$ ) range. Infrared and thermographic imaging have traditionally been used in specialized applications in military, mechanical engineering, satellite mapping, and energy efficiency testing. Millimeter-wave radiometric imaging has been used primarily in military and space applications.

The ability of radiation outside the visible spectrum to penetrate the atmosphere under conditions that would obscure normal visibility is of particular interest for traffic safety. There appears to be considerable interest in mechanisms for improvement of traffic safety during reduced visibility conditions. ${ }^{1}$ Since fog is a very common phenomenon on highways, intervention on the basis of visibility sensor data alone is neither practical nor advisable. Effective traffic management under visibilityrestricted conditions requires not only the ability to detect the presence of the visibility condition, but also the ability to assess the traffic situation.

\section{ATMOSPHERIC TRANSMISSION IN INFRARED AND MILLIMETER-WAVE SPECTRAL BANDS}

Atmospheric attenuation of image-forming radiation is produced by two physical mechanisms - absorption and scattering. Absorption is dependent on the frequency and the gases and particles present. Scattering is dependent on the size, shape, and dielectric constant of the scattering objects, and the wavelength of the energy scattered. Atmospheric constituents such as oxygen, water vapor, and carbon dioxide play a dominant role in determining millimeter-wave and infrared attenuation. The internal energy states of these molecules define frequencies at which the molecules absorb energy, thus creating frequency bands of high attenuation. Relatively low absorption exists in other intervals of the electromagnetic spectrum called transmission windows. Sensors that operate at these frequencies can propagate energy and the information contained in this energy over greater distances. Weather-related obscurants such as rain, snow, and fog add to the absorption and scattering experienced under clear weather conditions and further limit sensor performance. Window frequencies in the millimeter-wave spectrum occur at center frequencies of approximately $35,94,140,225$, and $350 \mathrm{GHz}$. Highway sensors that detect and classify objects at long ranges are typically designed for operation at these frequencies.

The infrared absorption spectra are due to molecular rotations and vibrations that occur in atmospheric molecules. The principle permanent atmospheric constituents contributing to the absorption of energy at infrared wavelengths are carbon dioxide, nitrous oxide, and methane. Variable constituents that absorb infrared energy include water and ozone. In addition to absorption, infrared energy is scattered from molecules and aerosols in the atmosphere. Wavelengths less than $2 \mu \mathrm{m}$ experience negligible molecular scattering. Scattering from aerosols is a function of the radius of the scatterer divided by the wavelength. Aerosol-type scatterers include rain, dust, fog, and smoke. The nearinfrared wavelength band, extending from 0.77 to $1.5 \mu \mathrm{m}$ is constrained at the upper end by water vapor absorption. The mid-infrared wavelength band from 3 to $5 \mu \mathrm{m}$ is bounded on the lower and upper ends by water vapor absorption. An absorption peak in the middle of the band is due to carbon dioxide. The far-infrared (or longwave IR) band extends from approximately 8 to 12 $\mu \mathrm{m}$. The lower wavelength is restricted by water vapor and the upper by a combination of water vapor and carbon dioxide.

The amount of infrared energy transmitted through a particular atmosphere (possibly containing humidity, fog, rain, or particulates) can be predicted by computer simulation programs such as LOWTRAN ${ }^{2,3,4}$ (LOW resolution TRANsmissivity simulation) that covers a spectral range of 0 to $50,000 \mathrm{~cm}^{-1}(0.2 \mu \mathrm{m}$ to infinity) with a resolution of $20 \mathrm{~cm}^{-1}$ full width at half maximum (FWHM). For the purposes of this study, we used an improved resolution version of LOWTRAN known as MODTRAN (MODerate resolution TRANsmissivity simulation) to estimate visibility through radiation and advective fogs.

\section{ADVANCED VIDEO IMAGING TECHNOLOGIES}

VISIBLE SPECTRUM IMAGING - Visible wavelength sensors, such as the human eye or CCD cameras, detect sunlight or artificial light that is reflected off objects. A prior comprehensive study of closed-circuit television (CCTV) technology for highway surveillance was completed in 1990-1992 by the authors at the Transportation Electronics Laboratory, California Polytechnic University at San Luis Obispo, CA ${ }^{567}$. Among the results of this study were observations that the most important performance factors for cameras 
used for highway surveillance are the resolution, dynamic range, and immunity to vertical or horizontal smear. ${ }^{8}$ The horizontal resolution generally correlates with the amount of detail resolvable from the video signal generated by the camera. For analog imaging devices and signals, this is synonymous with bandwidth. For solid state imagers such as CCD (Charge-Coupled Device) cameras, this is limited by the pixel density of the imaging array. Vertical resolution is fixed by the Electronic Industry Association $(E \mid A)^{9}$ or International Radio Consultative Committee (CCIR) specifications. As related to the needs of traffic surveillance, greater resolution implies that either (1) for a given angular resolution requirement, a larger area may be imaged, or (2) for a given image area requirement, a finer grain image may be discerned. Dynamic range and sensitivity relate respectively to the range of light levels than may be distinguished, and the ability of the imager to detect objects under low illumination conditions. Of the two factors, our prior experience has suggested that for most traffic surveillance situations, the dynamic range specification is of greater importance.

Closely related to dynamic range are the immunity of the imager to vertical or horizontal smear, which manifests as saturated vertical or horizontal bars in the image, originating from bright points (such as headlights) in the scene. Smear is attributed to extreme local saturation of the $C C D$, which causes charge bleed across all pixels in a column or row, depending upon the array topology.

INFRARED IMAGING - Infrared imaging utilizes spectral bands in which a strong image signature exists, atmospheric attenuation is minimal, and a detection method and optics are available for formation of an intensity-based image from radiation emitted by the scene. Related to the last of these considerations is the operational temperature requirement of the focal plane array or mechanically-scanned sensing element. Generally, the higher-performance solid state IR imagers require operation at cryogenic temperatures, typically 77 degrees Kelvin, while uncooled sensors of somewhat lower performance operate at room temperature $(\approx 298$ degrees Kelvin). Noise performance and sensitivity are the key performance differences between cooled and uncooled sensors. The cryogenic cooling requirement, however, makes camera technologies based upon cooled sensors more expensive and less reliable in terms of the mean time between failures (MTBF). Three common types of infrared detectors are available: quantum, microbolometer, and pyroelectric.

Quantum detectors, based upon the detection of individual photons, are used in a wide variety of applications because of their relatively high sensitivity and low noise, superior in both respects to microbolometers and pyroelectric detectors. All require cryogenic cooling for proper operation. Compound semiconductors typically form the basis of quantum detectors. Three types are currently in commercial use:
Platinum Silicide (PtSi), sensitive in the 3-5 micron IR band, Indium Antimonide (InSb), sensitive in the 3-5 micron IR band, and Mercury-Cadmium-Telluride (MeCdTe), sensitive in the 8-12 micron IR band. As will be described later, the majority of the IR cameras that we tested in this study employed quantum detectors. Refractive optics fabricated from Germanium or Silicon are required, which are transparent to infrared radiation in the respective bands.

Microbolometers require no cooling other than possible stabilization near room temperature. ${ }^{10}$. Unlike quantum detectors, the microbolometer array consists of pixels that measure temperature changes induced by incident infrared radiation. Minute temperature differences are sensed by changes in resistance as a function of temperature in individual pixels of a focal plane array. Sensitivity is maximum in the long wave infrared ( 8 to 14 $\mu \mathrm{m})$. band. Because microbolometer technology is monolithic, unlike laminated or bump-bonded photon arrays, it is rugged. Improvements in microbolometer technology are expected to bring increased sensitivity and lowered cost by keeping the current sensitivity and using less expensive optics. We tested only one camera based upon microbolometer technology, the GEC/ Marconi Sentry IR20. This camera uses an uncooled focal plane with a $200 \times 200$-pixel array sensitive to 8-14 $\mu \mathrm{m}$ radiation. The detector material used by this unit is lead zirconite $(\mathrm{PbZr})$. Because of the time-limited thermal mechanism underlying the detection process, bolometric imaging of full-motion video may be subject to image lag as well as analog video (e.g., vidicon) artifacts such as phenomena of flair, bloom and comet tail. ${ }^{5}$

The lowest cost technology for longwave IR imaging is the pyroelectric sensor. Uncooled pyroelectric arrays operate by measuring the change in the polarization of the detecting material induced by incident infrared radiation. Pyroelectric detectors usually consist of a thin layer of ferroelectric material sandwiched between two electrodes to form a capacitor. Since ferroelectric materials exhibit a spontaneous polarization that is temperature sensitive, the pyroelectric detector functions as a temperature-sensitive capacitor. They produce an output voltage proportional to the time rate of change of the temperature and not to the temperature itself. Signal processing translates these voltages into a representation of IR intensity from the scene. Although limited by thermal mechanisms, the response of pyroelectrics can be faster than that of microbolometers. Image noise appears to be the most significant performance limitation of this sensing technology. Two types of pyroelectric cameras were evaluated in this project, the Insight StarSight 8-14 $\mu \mathrm{m}$ and two versions (military and commercial) of the TI Nightsight C100 8-14 $\mu \mathrm{m}$ camera. The Insight IR camera is no longer in production, and it's detection physics could not be determined from the limited specifications in the operator's manual. Texas Instruments (TI) of Dallas TX, in cooperation with Hughes Aircraft Company of Los 
Angeles CA, uses a Barium Strontium Titanate (BST) focal plane in the Nightsight Camera. The focal plane contains 80,000 pixels $(245 \times 328)$ sensitive to the 8 to 14 $\mu \mathrm{m}$ IR band.

MILLIMETER-WAVE IMAGING - Imaging at radio frequencies is a more recent innovation, although the basic phenomena has been understood for decades. Passive millimeter-wave (MMW) sensors receive energy that is emitted or transmitted from other sources. In transportation applications, they may be used to detect and provide imagery of vehicles. Their longer wavelength (compared with infrared radiation) allows the reception and identification of energy emitted by an object virtually unaffected by fog, smoke, rain or particulates. Images may be formed using a single detection element with mechanical scanning of a highly directional antenna, or in a manner analogous to a CCD camera operating in the visible range. Respectively, radiation from the scene is either raster scanned or focused on a focal plane array via a reflective or transmissive optical element. When this signal is rendered on a video monitor, a visual-like image is produced.

A primary area of evaluation in our study was the relative ability of imaging technologies to penetrate atmospheric conditions that would otherwise obscure visible imaging. If the wavelength of observation for the millimeter-wave camera is chosen to correspond to one of the windows of high atmospheric transmission (for example, at 35 or 94 $\mathrm{GHz}$ ), atmospheric obscurants such as fog or smoke have little attenuating effect, and the images have striking contrast between the metal vehicle and the background. The contrast is due to specular reflection of downwelling sky radiation from the surface of the metallic vehicle to the sensor. At $94 \mathrm{GHz}$, the sky temperature is typically a few tens of degrees Kelvin. Rough, natural background textures tend to be highly emissive, and radiate energy at an effective temperature of about $300 \mathrm{~K}$, thus appearing to be much "hotter" than the metallic objects. Since the wavelengths are long compared with the size of suspended water droplets or other aerosols, the $94 \mathrm{GHz}$ image is unaffected by the presence off fog or particulates. Phenomenology and image properties are essentially unchanged between daytime or nighttime viewing.

Disadvantages for this type imaging system are the physical size of the scanner and fundamental resolution limitations related antenna theoretic considerations. 6 Image resolution is very low at the current level of MMW technology. The relative immaturity of the sensor hardware compared with infrared imagers must be noted. Hughes and TRW, among other firms, are currently developing passive millimeter wave imagers, ultimately intended for commercial applications. TRW is currently under contract to the DOD to produce a focal plane array millimeter-wave camera as an airport landing aid. ${ }^{11}$ The camera will operate at a center frequency of $90 \mathrm{GHz}$. The focal plane array of the camera will contain 1,040 monolithic millimeter-wave integrated circuit (MMIC) receiver chips, creating an $80 \times 52$ pixel image. Aperture deconvolution algorithms help display an image that refreshes 17 times a second. Given time and a significantly larger market, it is expected that the cost of a large pixel-count focal plane array will become more cost competitive.

\section{SELECTION OF EXAMPLES OF AVAILABLE IMAGING TECHNOLOGIES}

We attempted to study the characteristics and performance of examples of every advanced imaging technology currently available that could potentially be used for highway surveillance or automated detection. Manufacturers, developers, and vendors of equipment were identified and contacted. Imaging systems were acquired by loan, lease or purchase. Each system was tested under a range of atmospheric, illumination, and traffic scene conditions. ${ }^{11}$ The imaging systems tested are listed in Table 1. Purchase prices for the cameras tested range from a low of $\$ 8,000$ for the Texas Instruments NightSight, to a high of approximately $\$ 100,000$ for the Agema Thermovision 1000 . Since the scope of this study was restricted to technical considerations only, cost information is not stated for specific camera models.

\section{A SPECTRUM-INDEPENDENT METRIC FOR VIDEO IMAGE INFORMATION CONTENT}

Our objective was to comparatively measure the useful information content in video images obtained from imaging systems of completely different types. A preliminary literature survey identified related results in visual perception, but nothing directly applicable to this task. It was clear that whatever metric is applied would inevitably be application-specific, since information in an image is a relative concept, depending on the needs of the observer. A survey of traffic management professionals was conducted ${ }^{10}$, the results of which emphasized that useful visual information was most often based upon contrast, either in intensity or color, between a target and its background. Generally, the greater the contrast and cohesiveness of the target, the greater its detectability. Thus, a well-delineated light-colored car on a dark roadway surface has rich information content, while a less-delineated image of the car (for example, in fog or in a low-contrast IR) contains less useful information. Noise in the image, which manifests as random variations in pixel intensity or color, is considered "false" information. Ultimately, what a TMC operator needs to see (or classify, identify, assess) in a traffic scene are objects (vehicles, pedestrians, signage, equipment or debris on the roadway). 
Table 1. Imaging Systems Tested.

\begin{tabular}{|c|c|c|c|c|}
\hline $\begin{array}{l}\text { Company and } \\
\text { Product }\end{array}$ & $\begin{array}{l}\text { Received } \\
\text { Wavelength } \\
\text { Band }(\mu \mathrm{m})\end{array}$ & $\begin{array}{l}\text { Focal Plane } \\
\text { Temperature and } \\
\text { Cooler Type }\end{array}$ & Detector Type & $\begin{array}{l}\text { Array Size } \\
\text { (pixels) }\end{array}$ \\
\hline AGEMA Thermovision & 8 to 12 & $77 \mathrm{~K}$ Sterling & $\mathrm{HgCdTe}$ & $\begin{array}{l}5 \text { elements, } X-Y \\
\text { mechanical scan }\end{array}$ \\
\hline $\begin{array}{l}\text { Cincinnati Electronics } \\
\text { IRRIS-256ST }\end{array}$ & 3 to 5 & $77 \mathrm{~K}$ Sterling & $\operatorname{lnSb}$ & $256 \times 256$ \\
\hline FSI PRISM & 3.6 to 5 & $77 \mathrm{~K}$ Sterling & PtSi & $320 \times 244$ \\
\hline $\begin{array}{l}\text { GEC/Marconi Sentry } \\
\text { IR20 }\end{array}$ & 8 to 14 & Ambient & Microbolometer & $200 \times 200$ \\
\hline Inframetrics 600 & 3 to 5 and 8 to 12 & $77 \mathrm{~K}$ Cryogenic & $\mathrm{PtSi}$ and $\mathrm{HgCdTe}$ & $\begin{array}{l}1 \text { element, } X-Y \\
\text { mechanical scan }\end{array}$ \\
\hline Inframetrics 760 & 8 to 12 & $77 \mathrm{~K}$ Sterling & $\mathrm{HgCdTe}$ & $\begin{array}{l}1 \text { element, } X-Y \\
\text { mechanical scan }\end{array}$ \\
\hline Inframetrics InfraCam & 3 to 5 & 75 K Sterling & PtSi & $256 \times 256$ \\
\hline Insight/Starsight & 8 to 14 & Ambient & Pyroelectric BST & $256 \times 256$ \\
\hline Mitsubishi IR-M300 & 3 to 5 & $77 \mathrm{~K}$ Sterling & PtSi & $256 \times 256$ \\
\hline TI Nightsight & 8 to 14 & Ambient & Pyroelectric BST & $256 \times 256$ \\
\hline $\begin{array}{l}\text { TRW Multispectral } \\
\text { Scanner }\end{array}$ & $\begin{array}{l}94 \mathrm{GHz} \\
\text { (millimeter-wave) }\end{array}$ & Ambient & HEMT*-heterodyne & $\begin{array}{l}1 \text { element, } X-Y \\
\text { mechanical scan }\end{array}$ \\
\hline
\end{tabular}

* HEMT = high electron mobility transistor

Based upon these observations, the image analysis metric was developed which is similar to a signal-to-noise ratio, a commonly used measure of effectiveness for sensor or detector systems. In traffic management, the "signal" is contrast information in the image of a vehicle or other information object, with respect to the background road surface. ${ }^{12}$ The metric is referred to as an Information-to-Noise Ratio (INR). It is mechanized by computer-based analysis of digitized video images, acquired originally on S-VHS videotape, from groups of imaging systems concurrently viewing selected traffic scenes. The metric requires two sets of data. The first set is a series of video fields that contain only the background. The second set is a series of video fields containing information objects, in this case vehicles. These are referred to as the foreground set. Both sets of images are evaluated over identical sets of foreground fields.

The INR is defined as:

Information/Noise Ratio (INR) $=\frac{\text { Foreground Information }}{\text { Background Noise }}$ where the background noise is determined as

Background Noise $=\sum_{j=1}^{q}\left[\frac{\sum_{k=1}^{m}\left[B_{j}[k]-B K G[k]\right]^{2}}{m}\right]^{1 / 2} \cdot \frac{1}{q \cdot 255}$ $j$ is the video field index, $q$ is the total number of background fields, $k$ is the pixel index, and $m$ is the total number of pixels in each field. Each pixel can range in value from $0-255$, therefore the final result is divided by 255 to normalize the result to a range of 0.0 to 1.0 . $B K G[k]$ is the mean intensity of the $k^{\text {th }}$ pixel value across all $q$ background fields:

$$
B K G[k]=\frac{\sum_{j=1}^{q} B_{j}[k]}{q}
$$

$\left.B_{j} k k\right]$ is the $k^{\text {th }}$ pixel of the $f^{\text {th }}$ field in the set of background images.

The foreground information is found by examining selected field containing complete vehicle images. Over the selected set of video fields,

Foreground Info $=\sum_{j=1}^{n}\left[\frac{\sum_{k=1}^{m}\left[l_{j}[k]-\mathrm{BKG}[k]\right]^{2}}{m}\right]^{1 / 2} \cdot \frac{1}{n \cdot 255}$

Here $l[k]$ is the intensity of the $k^{\text {th }}$ pixel in the $i^{\text {th }}$ field of the foreground set, and $n$ is the total number of foreground fields. $n$ need not equal the total number of background fields $q$, since the foreground and background sets are normalized independently. 
The INR as defined above is independent of the resolution of the camera and the field of view, since it is normalized to the number of pixels in the image. It therefore measures the intrinsic imaging quality of a particular sensor technology rather than the performance of a particular imaging device. While it is generally desirable to be insensitive to the field of view, there are some situations in which the resolution of the camera, as well as the INR, are important considerations, such as the comparison of the performance of two competing products based upon a given technology. For these situations, a modified version of the INR is calculated by simply multiplying INR by the camera resolution in pixels. This is referred to as the INRR (INR-Resolution) metric. Both black-hot and white-hot infrared images are judged equally, since the metric is only effected by the magnitude difference in pixel intensities between the background and foreground. This also allows for a fair comparison between the intensity-based infrared images and black and white (intensity-based) visible images of the same scenes.

The above metric considers only intensity-based information in an image. Chromatic (spectral) information sensed by a conventional color video camera has been found to add substantially to the surveillance value of a video image. ${ }^{5}$ Color information can be incorporated in a modified form of the metric by summing the intensity information content of each of the three fundamental color components (red, green and blue) ${ }^{10}$. The same basic equation is used for each color component, and the result is summed. This approach recognizes that in a typical CCD color video camera, a "trixel" comprised of three detection elements (one for each color component) replaces the conventional pixel, so that three times the information is generated per unit of resolution. However, some of this information is redundant, such that the application of a color weighting factor $\mathrm{K}$ with value somewhat less than unity is appropriate on an application-specific basis.

False coloration is sometimes used to accentuate the contrast in imagery produced by infrared and millimeterwave cameras. Such images have been processed to replace intensity gradients with color gradients. This adds redundant information to the image. Ideally, the fullcolor INR metric should not be effected by artificial coloration of intensity-based images, and the color weighting factor $K$ may be adjusted to assure equivalence of the results for monochrome and false colored versions of the same.

The INR metric has distinct limitations that must be recognized if it is used for comparison of dissimilar imaging technologies. One consideration is the sensitivity of the metric to the size of the analysis window drawn in the digitized video field. This window is typically sized to be slightly larger than the size of typical vehicle, or approximately the width of a traffic lane in the scene. For valid comparisons between different cameras, it is important that the relative window size be the same for video produced by each. The metric is also sensitive to the selected test scene, such as the types and colors of the vehicles and the effects of shadows or other changing light conditions. It is therefore important that the same image sequences be used to generate the metric for each pair-wise camera comparison. All comparisons made in this study were produced from video acquired by groups three or four cameras viewing the same traffic scene concurrently. Linear SMPTE (Society of Motion Picture and Television Engineers) standard time code (LTC) was recorded on the audio tracks of a video tapes to assure frame-by-frame synchronization between all cameras in a comparison group. Comparative results for cameras from different groups are linked via a camera common to each different group, usually the color CCD camera. Overall comparison results are normalized to an arbitrarily selected single camera, by dividing all results by the results for this camera.

Because the millimeter-wave camera did not produce motion video, the metric was applied to a single foreground and background field of each scene. This limits the comparability of the results for the millimeterwave camera with other cameras. We note that the experimental TRW millimeter-wave camera we tested was designed for proof of concept only, and its results are not necessarily indicative of the ultimate potential of MMW imaging, which is expected to utilize focal plane arrays of detection elements based upon . recently developed MMIC (Monolithic Microwave Integrated Circuit) technology. ${ }^{13,14}$

\section{FIELD EVALUATION OF IMAGING DEVICES}

We evaluated videotaped imagery of traffic on California freeways, produced by each advanced imaging system shown in Table 1. These included ten infrared cameras and one millimeter-wave imager. One VNIR-modified CCD camera and one visible (color) reference camera (not shown in Table 1) were also included as reference devices in tests. All devices produced either EIA-RS170 or CCIR/PAL standard video signals. We sought to acquire imagery under a wide range of traffic, lighting, and atmospheric conditions. Ideally, test conditions would include combinations of traffic conditions (various levels of service and incidents), atmospheric conditions (clear, convection fog, radiation haze, rain, snow, smoke, dust), lighting conditions (clear mid-day sun, lowincidence angle glare, wet surface glare, snow glare, dusk/dawn low-light, darkness). A field test schedule was developed to implement as many test conditions as possible during periods of availability for each imaging system.

The test apparatus was comprised of five LTCsynchronized S-VHS video recorders, and a PC-based computer control system. We designed and fabricated a towable mobile surveillance trailer (MST) with a 10-meter collapsible camera tower to provide elevated camera positions in situations where freeway overcrossings were 
not available. A high-resolution color visible spectrum camera (Burle TC209) was used as a visible-spectrum reference camera, for a common point of comparison for all IR cameras under test. The apparatus supported the simultaneous recording and time-code synchronization of up to four imaging systems plus the visible reference camera.

Field tests were conducted during the period December 1, 1994 through May, 1995. A specially-equipped truck housed the video data acquisition systems and a mobile 110 VAC, $1.5 \mathrm{~kW}$ computer-grade inverter system for powering sensitive computer and video equipment. A small moving van was used to transport the collection of cameras and field apparatus to each test site. Approximately 160 hours of video tape and 100 digitized image files (from the millimeter-wave imager) were created at nine field sites in the Los Angeles, Long Beach, Fresno, the Central California Valley, Sierra Nevada Mountains, and San Luis Obispo county coastal areas. Caltrans District 6 permitted us the exclusive use of a new but unopened overcrossing at Fruit Avenue over Hwy 99 in Fresno.

Due to limited availability of the imaging systems, it was not possible to test all imaging systems at all locations or under all conditions. Although tests were conducted under a range of atmospheric conditions typical of light advective and radiation fog, only two infrared cameras (one 3-5 $\mu \mathrm{m}$ and one 8-12 $\mu \mathrm{m}$ ) were tested in visibility less than $0.1 \mathrm{~km}$. Visibility distance comparisons were based upon human observation of traffic cones placed at fixed distances. While valid for visibility measurements based upon FAA (Federal Aviation Administration) test standards, this visibility comparison method is of limited value for infrared cameras since the improved imaging capability of IR cameras is related to other radiation factors, most notably the surface temperature of the targets of interest, in this case, automobiles. Subject to the experimental limitations, our field observations appeared to support the trends predicted by MODTRAN for the visible, SWIR, LWIR, and $94 \mathrm{GHz}$ millimeter-wave bands.

Since the $94 \mathrm{GHz}$ millimeter-wave camera was capable of producing only still images acquired over a two-minute aperture time, it could not be used to view traffic scenes containing moving vehicles. This severely limited our ability to test it on an equal basis with the other video imaging systems. Many additional factors were found to influence the quality and information content of the IR, millimeter-wave, and visible images. These factors are related to differences in the information content of images in the IR and millimeter-wave bands as compared to the familiar visible imagery.

\section{ANALYSIS OF FIELD DATA FOR IMAGE INFORMATION CONTENT}

The normalized INR and INRR metrics were used to analyze and compare the imagery obtained from the infrared, visible, and millimeter-wave cameras. Table 2 lists specifications of the cameras evaluated. Columns 2 and 3 contain the horizontal and vertical resolutions and Column 4 the total number of pixels in the camera's focal plane. The fourth column contains the "resolution multiplier ${ }^{n}$ or pixel-quantity factor that converts the INR into INRR, that accounts for camera resolution. This factor is calculated by dividing the number of pixels in a camera's focal plane by 15,394 which was the lowest resolution focal plane array tested, the StarSight $8-14 \mu \mathrm{m}$ camera.

Table 2. Individual camera resolution and resolution multipliers.

\begin{tabular}{lllll}
\hline Camera & Horiz. Vert. & H x V & $\begin{array}{l}\text { Resolution } \\
\text { Multiplier }\end{array}$ \\
\hline Cincinnati Elect. 3-5 $\mu \mathrm{m}$ & 256 & 256 & 65536 & 4.26 \\
FSI Prism 3-5 $\mu \mathrm{m}$ & 320 & 244 & 78080 & 5.07 \\
TI NightSight 8-14 $\mu \mathrm{m}$ & 320 & 164 & 52480 & 3.41 \\
AGEMA 8-12 $\mu \mathrm{m}$ & 320 & 240 & 76800 & 4.99 \\
Burle Security Visible & 768 & 494 & 379392 & 24.65 \\
StarSight 8-14 $\mu \mathrm{m}$ (round) & 140 & 140 & 15394 & 1.00 \\
Inframetrics 600 8-12 $\mu \mathrm{m}$ & 194 & 240 & 46560 & 3.02 \\
Inframetrics 600 3-5 $\mu \mathrm{m}$ & 194 & 240 & 46560 & 3.02 \\
M300 3-5 $\mu \mathrm{m}$ & 256 & 256 & 65536 & 4.26 \\
Marconi 8-14 $\mu \mathrm{m}$ & 200 & 200 & 40000 & 2.60 \\
Inframetrics 760 8-12 $\mu \mathrm{m}$ & 194 & 240 & 46560 & 3.02 \\
Infracam 3-5 $\mu \mathrm{m}$ & 256 & 256 & 65536 & 4.26 \\
TRW Imager & NA & NA & NA & NA \\
\hline
\end{tabular}

NA = not applicable

Imaging performance is generally dependent upon lighting and weather conditions. Three categories of lighting conditions were used in these tests, night (very low light), day (good lighting), and dusk/dawn (low-tomedium light). Additionally, scenes containing significant fog were assigned three range/approach designators: close, far/approaching and far/departing. Close data refer to vehicles approximately $60 \mathrm{ft}$ from the camera, which characterizes the ability of a camera to image a vehicle in close proximity. The far data are divided into two categories, approaching vehicles and departing vehicles. Far approaching vehicles were approximately 400 meters ( $1 / 4$ mile) from the camera, which tested the ability of the camera to image a vehicle just as it leaves the fog, typically with headlights on, becomes visible to the human observer. Far departing vehicles were approximately 200 meters (1/8 mile) from the camera, proceeding into the fog bank, typically with taillights visible.

For ranking purposes, raw INR or INRR values of all cameras are normalized to that of the visible reference camera. If data from more than one session are to be compared, then there must be a common reference 
camera across all sessions. The cameras were tested in three groups: Group A contains the GEC/Marconi Sentry, Insight StarSight, Inframetrics 600, and Mitsubishi M300. Group B contains the Cincinnati Electronics Irris, FSI Prism, Texas Instruments NightSight, and the AGEMA Thermovision 1000. The Group C cameras consisted of the Inframetrics Infracam, Inframetrics 760 , and the Burle TC291. For cross-reference purposes, the AGEMA 1000 camera and Burle reference color camera were also deployed with the other groups. The AGEMA 1000 connected Group B infrared cameras with both Groups A and C. This approach yields a set of comparable results.

Tables 3, 4, 5, and 6 show normalized INR and INRR results for each camera across all data sets. Test conditions included: night/approaching traffic, night/ departing traffic, day/approaching traffic, day/departing traffic, dawn-dusk/approaching traffic, and dawn-dusk/ approaching traffic in fog. Data sets acquired in dense fog conditions have been separated into Tables 5 and 6 to obtain a more equal comparison. The GEC/Marconi camera used a PAL (Phase Alternating Line) European video format, which was converted to NTSC (National Television Standards Society) format for display and analysis purposes. This process introduced some additional noise. The TRW millimeter-wave imager is included in the no-fog camera sets of Tables 3 and 4. While not directly comparable with the IR and visible cameras, the millimeter-wave results generally indicate how the still image information provided in the $94 \mathrm{GHz}$ band compares with the other (full motion) imaging technologies tested.

Table 3. Normalized INR averages in absence of fog.

\begin{tabular}{|c|c|c|}
\hline Camera & $\begin{array}{l}\text { INR Normalized with } \\
\text { Respect to Visible Camera }\end{array}$ & Rank \\
\hline Cincinnati Elect. 3-5 $\mu \mathrm{m}$ & 0.345 & 2 \\
\hline FSI Prism 3-5 $\mu \mathrm{m}$ & 0.130 & 7 \\
\hline 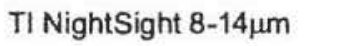 & 0.186 & 5 \\
\hline AGEMA $8-12 \mu \mathrm{m}$ & 0.248 & 4 \\
\hline Burle Security Visible & 1.000 & 1 \\
\hline 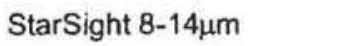 & 0.044 & 10 \\
\hline Inframetrics $6008-12 \mu \mathrm{m}$ & 0.136 & 6 \\
\hline Inframetrics $6003-5 \mu \mathrm{m}$ & 0.028 & 11 \\
\hline 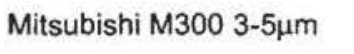 & 0.336 & 3 \\
\hline Marconi $8-14 \mu \mathrm{m}$ & 0.107 & 8 \\
\hline TRW Mulispectral Imager & 0.084 & 9 \\
\hline
\end{tabular}

Table 4. Normalized INRR (INR $\times$ resolution multiplier) in absence of fog.

\begin{tabular}{llc}
\hline Camera & $\begin{array}{l}\text { INRR Normalized with } \\
\text { Respect to Visible camera }\end{array}$ & Rank \\
\hline Cincinnati Elect. 3-5 $\mu \mathrm{m}$ & 0.060 & 2 \\
FSI Prism 3-5 $\mu \mathrm{m}$ & 0.027 & 5 \\
TI NightSight 8-14 $\mathrm{m}$ & 0.026 & 6 \\
AGEMA 8-12 $\mu \mathrm{m}$ & 0.050 & 4 \\
Burle Security Visible & 1.000 & 1 \\
StarSight 8-14 $\mu \mathrm{m}$ & 0.002 & 10 \\
Inframetrics 600 8-12 $\mu \mathrm{m}$ & 0.017 & 7 \\
Inframetrics 600 3-5 $\mu \mathrm{m}$ & 0.003 & 9 \\
Mitsubishi M300 3-5 $\mu \mathrm{m}$ & 0.058 & 3 \\
Marconi 8-14 $\mu \mathrm{m}$ & 0.011 & 8 \\
TRW Mulispectral Imager & $\mathrm{NA}$ & $\mathrm{NA}$ \\
\hline
\end{tabular}

NA $=$ not applicable

Table 5. Normalized INR averages in dense fog.

\begin{tabular}{lll}
\hline Camera & $\begin{array}{l}\text { Average INR Normalized } \\
\text { with Respect to Visible } \\
\text { Camera }\end{array}$ & Rank \\
\hline Burle Security Visible & 1.000 & 2 \\
Inframetrics 760 8-12 $\mu \mathrm{m}$ & 0.248 & 3 \\
Infracam 3-5 $\mu \mathrm{m}$ & 1.408 & 1 \\
\hline
\end{tabular}

Table 6. Normalized INR (INR $x$ resolution multiplier) in dense fog.

\begin{tabular}{lll}
\hline Camera & $\begin{array}{l}\text { Average INRR Normalized } \\
\text { with Respect to Visible } \\
\text { Camera }\end{array}$ & Rank \\
\hline Burle Security Visible & 1.000 & 1 \\
Inframetrics 760 8-12 $\mu \mathrm{m}$ & 0.046 & 3 \\
Infracam 3-5 $\mu \mathrm{m}$ & 0.243 & 2 \\
\hline
\end{tabular}

When using these results to compare the relative ability of each imaging technology to "see through" atmospheric aerosols such as fog, the original source of the scene illumination must be considered. In all fog test scenes, natural illumination was used. This means that the metric considered only one-way transmission of radiation from surfaces in the scene to the camera. When the source of scene illumination is coaxial or nearly coaxial with the 
path from the target to the imager, the effects of source backscatter can profoundly reduce the image information content. Backscatter tends to follow forward diffusion (a transmissivity-reducing factor), so that useful information content in the image declines in an approximate square-law manner with fog density. Backscatter disproportionately degrades visible spectrum imaging, especially at night, while having little or no effect in spectral bands for which black-body radiation is the predominant scene illuminant. Therefore, the results for visible spectrum imaging in fog are applicable to natural illumination situation only (from daylight or headlights of vehicles in the scene) and could be significantly worse in any of a number of artificial illumination scenarios. We justified this limit of testing by noting that most existing Caltrans surveillance cameras rely upon natural scene illumination or artificial illumination sources not proximate to the camera position.

\section{CONCLUSIONS}

The camera rankings shown in the tables can, in most cases, be anticipated from human observation of the video imagery on a monitor. Our reduced visibility field data was limited to light radiation fog and light-tomoderate advection fog conditions. We did not acquire enough comparative imagery in dense fog conditions to thoroughly evaluate the relative performance of the different spectral bands under these conditions, nor did we evaluate the relative effects of source backscatter on image degradation.

Based upon the INR, for situations without fog, the visible camera performs best, followed by the 3-5 $\mu \mathrm{m}$ cameras. Under fog conditions, the 3-5 $\mu \mathrm{m}$ camera generated the best results, with the visible camera still providing acceptable imaging results. The relatively poor INR results for the long wave IR cameras we initially found surprising in view of the significantly greater atmospheric transmission of long wave IR radiation compared with visible. This appears to be attributable to the significant value of the chromatic information available in the visible spectrum, and the headlight information available in the visible, VNIR and 3-5 $\mu \mathrm{m}$ IR bands, each of which contributed substantially to detectability of vehicles in fog. These factors more than compensated for the reduced transmissivity in these bands. Under fog conditions, the INRR results (which include the effects of image resolution) for the visible color camera were highest by a factor of approximately four. However, these results apply only to natural scene illumination, and it is known that source (e.g., headlight) backscatter could significantly reduce the usability of visible spectrum imaging in fog.

The longwave IR (8-12 $\mu \mathrm{m})$ and millimeter-wave (94 $\mathrm{GHz}$ ) bands have some intrinsic advantage under combined conditions of darkness and fog, not only due to improved transmissivity, but because images formed in these bands are based almost exclusively upon blackbody emissions of objects in the field of view.
Longwave IR images appear to be virtually immune to headlight or streetlight backscatter effects. With shorter IR wavelengths, specular effects begin to dominate, and this advantage is gradually lost. For example, mid-wave IR daylight images often contain infrared shadows, similar to visible spectrum shadows. The characteristics of VNIR images are so close to those of monochromatic visible spectrum images, that there appears to be no identifiable advantage for traffic monitoring, other than possibly covert surveillance with artificial VNIR illumination.

The $94 \mathrm{GHz}$ passive millimeter-wave camera detected warm bodies (human, vehicular or other) against a cold background or sky. Smooth metallic surfaces "reflected" the cold sky, giving them a dark appearance for a whitehot type of image. This imager produced only still images of such poor resolution as to be of little surveillance value despite the exceptional fog-penetrating characteristics of this spectral band. However, this result is considered not representative of the ultimate capabilities of the technology, but is attributed to the experimental limitations of the system that we tested.

The information content of infrared images is significantly different than that of visible spectrum imagery. These differences generally affected our subjective sense of the quality of the imagery, especially if no special consideration was given to the unique value of the additional information available in the IR images.

In the 8-12 $\mu \mathrm{m}$ longwave IR band, the windshield appears opaque and the engine, tire, and exhaust signatures appear more prominent, as detection in this band responds almost exclusively to heat emissions. IR reflections from pavement (such as reflected engine radiation) are strong in midwave $I R$ images and somewhat weaker in longwave images. In longwave IR, solar shadows cannot be detected, although slight differences in pavement temperature, such as on surfaces below an overcrossing, are clearly evident. Solar IR shadows are also evident in the mid-wave IR band, but pavement temperatures are less detectable.

The characteristics of images in the very near infrared (VNIR) band images are similar to those of monochrome visible spectrum images, such that there appears to be no discernable advantage to using this band for traffic monitoring, except perhaps covert surveillance using artificial near infrared illumination.

Field observations generally correlated with MODTRAN transmissivity predictions for clear weather and fog. It was not possible to differentiate between advective and radiative fog conditions in the field tests.

Overall, there appears to be a limited number of situations for which non-visible spectrum imaging appears to be justified. Based on information-to-noise measurements, we conclude that, except for a limited number of special situations, infrared and millimeterwave imaging technologies provide marginal or no net advantage compared with conventional color $C C D$ video 
cameras for typical surveillance needs. Specialized situations that may warrant the use of IR or millimeterwave imaging seem to be constrained to the following:

1. Roadway situations characterized by frequent dense fog, smoke or dust, in combination with recurrent hazardous traffic patterns, where surveillance and intervention by TMC personnel could reduce traffic incidents or loss.

2. Situations in which temperature information in the scene is useful, for example, detection of overheated truck brakes for HOV inspection.

3. Machine vision applications in which consistent scene illumination is critical, or the rejection of shadows and/or glare are required for proper detection or measurement.

It is important to recognize the immature state of these technologies compared with consumer and commercial video camera technology. Cooled detector technologies are now, and will probably remain too expensive and sensitive to consider for roadway surveillance. However, the present cost of uncooled IR focal plane arrays is primarily related to invested engineering and process considerations, such that significant reductions in cost and improvements in performance can reasonably be expected with higher production volumes.

\section{ACKNOWLEDGMENTS}

Support for this study was provided by the California Department of Transportation and the California PATH Program. Several graduate and undergraduate research assistants, including Jan Nimic, Brian Mealy, Matt Cotton and Cynthia Richardson contributed significantly to the field work and data reduction for this study.

\section{REFERENCES}

1. F. D. Shepard, Synthesis of Highway Practice 228: Reduced Visibility Due to Fog on the Highway, Transportation Research Board, National Academy Press, Washington, D.C., 1996.

2. Kneizys, F.X., Shettle, E.P., Gallery, W.O, Chetwynd, Jr., J.H., Abreu, L.W., Selby, J.E.A., Fenn, R.W., and McClatchey, R.A., Atmospheric Transmittance/ Radiance: Computer Code: LOWTRAN 5, AFGL-TR80-0067, AFGL, Hanscom AFB, MA 01731, 1980.

3. Kneizys, F.X., Shettle, E.P., Gallery, W.O, Chetwynd, Jr., J.H., Abreu, L.W., Selby, J.E.A., Clough, S.A., and Fenn, R.W., Atmospheric Transmittance/Radiance: Computer Code LOWTRAN 6, AFGL-TR-83-0187, AFGL, Hanscom AFB, MA 01731, 1983.

4. Kneizys, F.X., Shettle, E.P., Abreu, L.W., Chetwynd, Jr., J.H., Anderson, G.P., Gallery, W.O., Selby, J.E.A., and Clough, S.A., Users Guide to LOWTRAN 7. AFGL-TR-88-0177, AFGL, Hanscom AFB, MA $01731,1988$.
5. MacCarley, C.A., Evaluation of Closed-Circuit Television Technology for Application in Highway Operation, TR 91-4, Final Report, Rev. 2, California Polytechnic University at San Luis Obispo, CA, June 30, 1992.

6. MacCarley, C.A., Need, D. and Neiman, R. "Video Cameras for Roadway Surveillance: Technology Review and Product Evaluation Results," Transportation Research Record No. 1410, National Research Council, Washington D.C., 1993.

7. MacCarley, C.A.. and Ponce, L. "Video Technologies for Roadway Monitoring and Automated Detection", Society of Automotive Engineers, International Congress and Exhibition, Detroit, MI, 1996.

8. MacCarley, C.A., Study of Advanced Image Sensing Methods for Traffic Surveillance and Hazard Detection: First Year Progress Report, MOU 14, PATH Report 97-C20, California PATH Program, University of California, Berkeley, April 1997.

9. Benson, Blair K. ed., Television Engineering Handbook, McGraw Hill, New York, 1986.

10. Kreider, J., P. Howard, C. Li, and T. Fitzgibbons, "Uncooled infrared arrays sense image scenes," Laser Focus World, Aug. 1997, pp. 139-150.

11. MacCarley, C.A., "Advanced Imaging Techniques for Traffic Surveillance and Hazard Detection," Intellimotion, Vol. 6, No. 2, 1997, pp. 6-9.

12. MacCarley, C.A., B. Hemme, and L.A. Klein, Advanced Image Sensing Methods for Traffic Surveillance and Detection. Document No. TEL5859F98 (Rev 1), California Polytechnic University, San Luis Obispo, CA, June 30, 1998.

13. McHale, J., "TRW develops weather-piercing camera," Military \& Aerospace Electronics, July 1997, Vol. 8, No. 7, p.1.

14. Carts-Powell, Y., "Passive millimeter wave imaging growing fast," OE Reports, March 1997, p. 2. 